\title{
Representaciones sociales en las elites empresariales de la ciudad de Lima, Perú
}

\author{
Social representations in the economic elites \\ of the city of Lima, Peru
}

\author{
Rafael Baldomero López Lozano \\ Universidad Nacional Mayor de San Marcos \\ lopezlozanorafael@gmail.com \\ Código Orcid: https://orcid.org/0000-0002-0316-3695
}

\begin{abstract}
Resumen
El presente artículo expone las representaciones de los actores sociales que componen la nación entre un grupo de empresarios y ejecutivos pertenecientes a diversos gremios empresariales de Lima. En ese sentido, el trabajo recoge una serie de narrativas de los entrevistados en donde hacen un recorrido entre su trayectoria personal junto con el contexto que les tocó vivir. De esa forma, el objetivo del presente estudio es compartir las representaciones de las elites económicas más desde la narrativa y el testimonio que desde la estructura.

Estas representaciones giran en torno a la representación sobre empresarios, el Estado y distintos grupos sociales. Así, la investigación nace de la necesidad de estudiar a los sectores más privilegiados desde la antropología porque ayuda a entender como en las sociedades latinoamericanas se ejerce el poder. Por ese motivo, exponemos primero el caso de estudio, la metodología empleada y los estudios realizados sobre elites económicas en el Perú.
\end{abstract}

\section{Palabras clave}

Representaciones, elites económicas, nación, narrativas.

\begin{abstract}
This article exposes the representations of the social actors that make up the nation among a group of businessmen and executives belonging to various business associations in Lima. In that way, this work recollected narratives of their personal lives inside the social context. For that reason, the objective of these research was share the economic elite representations in the perspective of narratives and testimony instead of structure studies.

These representations were around businessmen, the government and different social groups. In addition of this, the research is born from the need to study the most privileged sectors from anthropology because it helps to understand how in Latin American societies power is exercised. Finally, we exposed the case of study, the methodology used and the researches about economic elites in Peru.
\end{abstract}

\section{Keywords}

Representations, economic elites, nation, narratives.

Forma sugerida de citar: López Lozano, R. (2019). Representaciones sociales en las elites empresariales de la ciudad de Lima, Perú. Universitas, 31, pp. 141-157. 


\section{Introducción}

El presente artículo tiene como objetivo exponer las representaciones de los actores que componen la nación entre empresarios y ejecutivos de Lima. Estas fueron desarrolladas dentro de un capítulo de la tesis "Análisis del discurso sobre la sociedad peruana en los principales gremios empresariales de Lima" presentada en la UNMSM en abril del 2019. Ella se enmarca dentro de la necesidad de estudiar el sector empresarial desde la antropología porque implica entender como dentro de las sociedades latinoamericanas se ejerce el poder.

La información fue obtenida luego de una serie de conversaciones con empresarios y ejecutivos afiliados a distintos gremios empresariales de Lima, Perú. En ellas, se pidió a los entrevistados que hagan un paralelo entre sus vidas profesionales junto con el contexto político que les tocó vivir y desarrollen una narrativa. Así, pudo conocerse cómo conciben a la nación peruana y las representaciones de otros grupos los dirigentes de los principales gremios económicos a nivel nacional a inicios del presente siglo.

El documento está divido en tres partes. La primera brinda la información general sobre el caso de estudio, describiendo la metodología empleada y brindando datos contextuales del grupo para ubicar al lector. Después, se revisan los estudios que han tratado sobre empresarios y nación en el Perú, componiéndose de una revisión bibliográfica sobre elites económicas, así como de qué se habla cuando nos referimos a reflexividad, narrativas y nacionalismos con el propósito de brindar herramientas analíticas. Y en tercer lugar exponemos lo dicho en las conversaciones en cuanto a representaciones sobre tres tópicos: Estado y políticos, empresarios, y representaciones sobre los distintos movimientos sociales con los cuales interactuaron.

Quiero finalmente mencionar que consideramos que la antropología tiene mucho que decir sobre los grupos más privilegiados de nuestras sociedades. Esto porque cuenta con una metodología cualitativa capaz de comprender al actor. Así, el propósito de este artículo es abrir una agenda de investigación que permita realizar nuevos aportes a la disciplina.

\section{Caso de estudio: los empresarios limeños}

En este trabajo, se conversó con diversos miembros del sector empresarial limeño para conocer sus trayectorias profesionales dentro del contexto social que les tocó vivir. Algunas pueden ser calificadas como historias de 
vida y otras como entrevistas en profundidad si tomamos en cuenta el tiempo de duración. Compartimos así los rasgos más generales de los entrevistados para brindar al lector características que le ayuden a contextualizarse.

Fueron realizadas en total veinte entrevistas a empresarios y ejecutivos. La duración de cada una dependía de la dinámica, donde algunos temas se profundizaban. Todos los entrevistados viven en Lima, siendo las conversaciones realizadas mayormente en los distritos de San Isidro, Miraflores y Surco. En cuanto al tipo de lugares, estos fueron cafés, oficinas y viviendas particulares.

De los 20, seis pertenecen a la industria, cuatro al comercio, cuatro a minería y el resto están en pesca, agricultura y marketing. En industria, se cubrieron las ramas de textiles, prendas de vestir, metalmecánica, bebidas y alimentos. Después, en el lado comercial, la mayoría de las personas pertenecientes a este sector ocuparon cargos gerenciales.

Tomando como referencia la propiedad, existen dos grupos: quienes están ligados a la propiedad de las empresas y los gerentes encargados del gobierno corporativo. La ubicación que ellos tienen dentro de ella incide en el tipo de decisiones y las capacidades para llevarlas a cabo. Por otro lado, los dueños son considerados aquellas personas que toman las decisiones finales. Ellos o su familia mantienen la propiedad y el control de una empresa en última instancia.

Dependiendo del caso, el control es delegado a un tercero para la administración de su negocio. Como comenta una entrevistada, ellos son considerados los verdaderos empresarios. Tenemos que recordar entonces que existe una relación muy cercana entre ambos grupos, siendo hasta miembros de la misma familia.

Los ejecutivos son toda persona empleada profesionalmente que ocupa los cargos más elevados en la toma de decisiones al interior de un esquema de gobierno corporativo. Ellos no tienen propiedad de la misma, son contratados por los dueños, pero se forman profesionalmente para administrar. Eso se refleja en, por ejemplo, que todos los ejecutivos entrevistados cuentan con maestrías y diplomados en Alta Dirección.

Respecto a sus centros de estudios, la mayoría asistieron a universidades privadas de Lima o en un College de Estados Unidos. También hay otro grupo que estudió en instituciones públicas como la Universidad Nacional de Ingeniería. Si bien todos son licenciados, un grupo interesante cuenta con estudios de maestría, obtenido tanto en Estados Unidos, Europa o América Latina. 
Y, por último, respecto a sus vínculos con el sector público, 5 de los 20 entrevistados ocuparon altos cargos desde los periodos de Alberto Fujimori (1990-2000) hasta Ollanta Humala (2011-2016). Después, 2 entrevistados forman parte de estudios de abogados que colaboraron con el Estado en modificaciones de leyes y privatizaciones. Además, existe un grupo que destaca por su experiencia tanto en el sector privado (empresas y gremios) como en el sector público. Ellos, más que pertenecer a determinadas asociaciones profesionales o cámaras, han sido formados en determinadas universidades en las carreras de derecho, economía, administración e ingeniería industrial. Es decir, su espíritu de cuerpo nace de la trayectoria educativa.

En ese sentido, el criterio de selección de los testimonios se basó en la búsqueda de la mayor diversidad de perspectivas posibles tomando en cuenta trayectorias profesionales. También se pensó en características generales como edad, sexo o sector que, consideramos, influye notoriamente en la formación de las representaciones. Por otro lado, cuando hablamos de las empresas, nos referimos a las principales compañías de capitales peruanos en su sector a nivel nacional. Esto junto a ejecutivos que han realizado su línea de carrera en empresas multinacionales. Así, tanto estas características de tipo demográfico como la bibliografía brindada a continuación ayudarían a comprender las representaciones encontradas.

\section{Elites económicas y nación}

\section{Estudios sobre elites económicas en el Perú desde las ciencias sociales}

La importancia de conocer los estudios que trabajaron sobre empresarios y nación se basa en observar cómo otros investigadores hablaron del tema asumiendo que toda investigación debe rescatar lo trabajado por otros investigadores. Este tema no es nuevo, sino que siempre ha estado presente en la tradición intelectual peruana. En el caso de las ciencias sociales, ha sido recurrente pensar sobre cómo piensan los sectores más privilegiados.

De acuerdo a lo encontrado, las investigaciones sobre los empresarios y ciencias sociales en el Perú tuvieron un propósito militante en sus inicios. El objetivo en ese entonces fue reconocer la naturaleza de la clase dominante 
para con ello delimitar una estrategia revolucionaria (Malpica, 1964). En ese sentido, se asoció empresarios con oligarquía porque tenían riqueza y poder (Cotler, 1978). Al estar constituida por grupos familiares, se formó la idea de una oligarquía con una posición dominante pero no dirigente (Rénique, 2008).

Con el fin del gobierno militar (1980) ya no fue la oligarquía sino el término de grupo económico para estudiar a los empresarios (Alcorta, 1989). Ello por la influencia de los departamentos de economía, que buscaban estudiar el desarrollo de su riqueza. Esto ocurre entre finales de los ochenta y principios de los noventa, coincidiendo con la introducción de reformas neoliberales en nuestro país (Anaya, 1990). Así, el objetivo fue conocer el impacto de estas políticas económicas en la vida económica peruana.

Después, durante el nuevo milenio se desarrollan nuevos temas, que van desde las relaciones de género hasta las redes de lobby informal (Ramos, 2014). Esto porque los grupos empresariales son vistos como parte del sector más privilegiado de la sociedad: una "clase alta" (Kogan, 1999). De manera general, el problema central abordado en las investigaciones fue el impacto de las desigualdades tanto económicas como políticas y sociales, así como a la captura del Estado por parte del poder económico (Campodónico \& Durand, 2018).

Luego están los estudios elaborados por las mismas asociaciones empresariales. Si bien en muchos casos son de tipo laudatorio, estos constituyen un aporte valioso porque muestran un relato sobre el rol cumplido por los empresarios en la construcción de la nacionalidad peruana (Reyes, 2008). Cabe resaltar que la mayoría de estos trabajos fueron realizadas en dos lugares: 1. Universidades de "elite" como la Universidad del Pacífico o la PUCP y 2. ONG dedicadas al estudio sobre desigualdades (Durand, 2016).

Por ende, apreciamos que el tema de nación se encuentra de forma indirecta en la mayoría de estos trabajos. Si bien su objeto central de estudio fue el ejercicio de poder desde o para el Estado, dejan algunas ideas sueltas sobre su visión de sociedad. Esta implica la representación de una comunidad política, que es la nación. Así, pensar en nación constituye un primer paso para trabajar sobre las representaciones.

\section{Reflexividad, narrativas y nación en la antropología}

La importancia de hablar sobre reflexividad, narrativas y nación radica que son categorías útiles para comprender cómo las representaciones son 
parte de una forma de concebir el mundo. Esto es, brindar categorías de referencia que provean de un soporte conceptual a la investigación empírica. Así, antes de hablar sobre lo dicho por los entrevistados, debemos comprender que, en la vida social cotidiana, nace la reflexividad del actor y esta surge como resultado de ella.

En los grupos sociales, junto con sus esquemas de percepción y apreciación, hay una lucha al interior del espacio social para imponer una determinada visión del mundo (Bourdieu, 2011). De acuerdo con nuestro tema de estudio, los entrevistados generan sus percepciones en gran medida gracias a la interacción que ofrecen los gremios como espacios de socialización y producto de sus propias historias personales, formando un "espíritu de cuerpo".

En ese sentido, debemos apreciar la necesidad de las narrativas puesto que forman parte de cómo un grupo social ve el mundo y sobre este actúa (Jimeno, 2016). Estas deben ser estudiadas de acuerdo al significado de los mensajes que buscan transmitir los sujetos dentro del lugar en donde se enuncian los discursos, tomando en cuenta cómo las categorías y subcategorías se relacionan. Por ende, las narrativas son una forma de ver la realidad social por parte de los entrevistados que, por su relación con el Estado, ha devenido en hegemónica porque se ha convertido en sentido común al interior de todo el sector empresarial agremiado y es transmitida en los medios de comunicación social, dando la idea de sentido común (García-Huidobro, 2016).

Una de las formas en cómo se manifiesta esta narrativa hegemónica en el sentido de Gramsci que (tiene que ver con el discurso sobre nación. Esto porque articula su discurso de forma coherente en el devenir de la nación, reuniendo en el relato a otros grupos sociales fuera de los empresarios y sectores de la sociedad peruana entorno a un discurso común sobre la realidad social. De esa forma, la narrativa sobre nación es importante porque constituye una manera de ganar legitimidad frente a otros grupos para tener mayor liderazgo en la toma de decisiones públicas.

Es útil hablar de nación porque esta lleva a pensar a la comunidad política y el rol que cumplen los entrevistados en su formación. Por otro lado, la influencia de las representaciones reside en que se vuelven parte de la forma en cómo los actores conciben su comunidad, siendo parte de la narrativa. Hablar de nación nos ayuda a comprender las formas de pensar el manejo de la sociedad y los tipos de liderazgo que pueden existir, entendiendo mejor las relaciones de poder. 
La nación y los nacionalismos son así una comunidad imaginada (Anderson, 1993) que nace dentro del contexto de la formación de la economíamundo (Wallerstein, 2006) y la aparición de la política de masas (Hobsbawm, 1997). Este debe estudiarse desde la reflexividad de los agentes, así como en la construcción de narrativas dentro de un tiempo heterogéneo, en cual las narrativas históricas son llenadas de contenido y donde hay un contenido surgido de las relaciones de poder (Chatterjee, 2002).

En el Perú, de acuerdo a lo encontrado, los grupos más privilegiados no desarrollaron un programa político y tampoco tuvieron un proyecto en torno al cual aglutinar a las otras clases, por eso tampoco se preocupó por constituir un grupo orgánico de intelectuales que ayudaran a su dominación de clase (Burga \& Flores, 1980). Se terminó construyendo, entonces, una imagen mitificada de la historia peruana en la que se exaltaba los elementos hispánicos, pero más como una forma de ver el mundo poco consciente. A nivel general, el Estado peruano se sustentó en las relaciones de clientela que los diferentes grupos oligárquicos tenían establecidas tanto con la población subordinada como con el capital extranjero (Cotler, 1978).

De esa manera, la nación puede verse como una comunidad imaginada que se contextualiza dentro la economía mundo. En paralelo, la política de masas genera un nacionalismo popular, que debe ser analizado desde la perspectiva del demos (comunidad política) y ethnos (comunidad cultural), instándose en la reflexividad de los agentes (Balibar, 2003). Esta constituye una de las formas de manifestación de las representaciones de los otros grupos sociales. Por ello, en paralelo a la historia de los nacionalismos debe existir una historia de la historia de los nacionalismos (Palti, 2003) porque implica una construcción y reconstrucción de los grupos sociales desde las elites, atribuyéndoles características y funciones dentro de la una comunidad imaginada.

En conclusión, es posible afirmar lo siguiente. 1) Las personas generan sus esquemas de percepción y apreciación a causa de su interacción en la vida social cotidiana. 2) Esos esquemas de pensamientos nacidos de la reflexividad va generando diversas concepciones de la realidad social. 3) La nación y el nacionalismo es una forma determinada de esos esquemas de representación de una colectividad que giran en torno a la comunidad política y la comunidad cultural, la cual es construida y atravesada por relaciones de poder. Por ende, estas categorías ayudan a entender que los actores se conciben como parte de una comunidad mayor, en este caso nacional, y forman un discurso que les permita integrarse a ella. 


\section{Resultados de la investigación}

\section{Estado y políticos}

En primer lugar, lo público es visto como burocrático, demora y necesita corrupción para acelerarse. Algo común sobre los políticos, funcionarios públicos y burócratas es que son despreciables porque se asume mediocridad de esa persona al no ser capaz de formar algo de manera independiente. En la mayoría de relatos, se retrata al Estado como poco confiable, falto de seriedad e incapaz de entender la complejidad del mundo corporativo.

Para los entrevistados, las relaciones con el Estado se personifican la relación en términos de quien ocupa su jefatura. Es decir, no se puede hablar de una "relación con el Estado" sino de las relaciones con determinados gobiernos y personas. Esto se encuentra particularmente explicado por la necesidad de los gremios empresariales de estar cerca del poder. En ese sentido no existe una relación con el Estado sino con el gobierno de x o z.

Tomando en cuenta esto, recuerdan más las relaciones con las empresas y el gobierno de acuerdo con tres periodos. En ese sentido, las principales etapas fueron el gobierno militar (1968-1980), la crisis del gobierno aprista (1987-1990) y el régimen de Fujimori (1990-2000). Cada una de ellas tiene una representación particular porque implicó una relación totalmente distinta una respecto a la otra.

El periodo del gobierno militar fue visto como mal organizado y prepotente. Por otro lado, el gobierno aprista es recordado como de crisis económica y terrorismo. Finalmente, el gobierno de Fujimori es visto con ambigüedad: se elimina el terrorismo pero se termina corrompiendo con la corrupción y el fraude electoral.

Particularmente, hay una especial representación con los políticos que se definen de "izquierda". Si bien podría asociarse con resentimiento social, más fuerte es la percepción de estos como antipáticos y que se alimentan del ambiente universitario porque su discurso crítico es atractivo cuando uno es joven. La idea central sobre sus propuestas es la siguiente: hay una visión idealista y poca práctica de la realidad, por ende terminan generando más problemas.

La crítica más fuerte es su doble discurso: hablan mucho de la necesidad de igualdad económica entre las personas, pero son los primeros en ostentar lujos. En ese sentido, dicen una cosa, pero hacen otra. Finalmente, todos 
consideran que el comunismo fracasa porque cuando no hay diferencia entre el trabajador y el ocioso, el trabajador no trabaja.

Si bien hablan de otros partidos políticos, la mayor importancia se le da a la izquierda. Esto por considerar que se promueve el resentimiento y el conflicto. En ese sentido, un giro radical es pensado como algo que atente contra la propiedad de las personas. Por otro lado, se asume que esto ocurre porque la izquierda peruana nunca ha llegado al Gobierno.

\section{Empresarios, sectores y gremios}

Dentro de las características de todos los empresarios, se lo asocia como una persona luchadora que genera riqueza real para su comunidad. Puede haber diferenciaciones, pero es a nivel general, una persona pragmática que quiere ganar dinero de forma legal y en la medida que cumple sus ambiciones, hace bien a la sociedad en general.

En las conversaciones sostenidas, la idea más frecuente refiere a las empresas como generadores de riqueza y los gobiernos como generadores de pobreza. En el fondo, "dar trabajo" les permitiría ganar legitimidad frente a otros actores y posicionarse como el agente que debe tomar el liderazgo en la toma de decisiones, siendo sus criterios los más adecuados. Ello

Empresario es, entonces, definido como quien realiza un negocio lícito, genera riqueza y trabajo. En el proceso produce transformación material, cultural, social y económica. Su modo de hacer las cosas es pragmático porque está todo el tiempo buscando la forma de solucionar problemas.

Generalmente se piensa que su deber es hacer empresa, buenos productos para vender, ganar dinero honradamente y en el camino genera empleo. Con eso, ya hace bien a la comunidad. De esa forma, cree que cumpliendo con su trabajo está contribuyendo a la comunidad. Sin embargo, esta puede ser circunstancial y muy dependiente del contexto.

Después, existe la idea generalizada que el empresario peruano hace cárteles por medio de los gremios empresariales y los estudios de abogados, oligopolios para defenderse mutuamente y competir bajo ciertos márgenes. Este vive regularmente en el corto plazo porque está atrincherado, pensando en evitar el ataque del gobierno. Además, existe una lenta transformación del sector empresarial en el Perú debido a un cambio generacional. Esto porque el mundo empresarial se ha complejizado y conforme más grande es una 
empresa, se va haciendo cada vez más difícil conocer los actores que toman las decisiones.

Además, caracterizan la forma de organizar el mundo corporativo como jerárquica, donde las cabezas mandan. En toda compañía, como en las familias, tiene que haber un orden para que puedan tomarse las mejores decisiones. Ella marca la pauta de la compañía, los objetivos y todos los recursos humanos deben empujar a esos objetivos. Por esa capacidad de tomar decisiones es que ganan el dinero que gana.

La principal diferencia entre los empresarios se da en el sector donde uno se encuentra trabajando, los gremios empresariales y la generación. Así, las representaciones, como hemos visto, parten de experiencias cotidianas de la vida social. En esa línea, están los empresarios "liberales", que, al contrario de los proteccionistas, son más ambiciosos porque querían salir a mar abierto, pescar los peces grandes. Quien se identifica con esta idea, serían las personas que de verdad piensan el país, a diferencia de un proteccionista que simplemente ve sus intereses.

Un sector que se autodefine como particular es el industrial. Este se representa como innovador, aunque muchos empresarios están poco dispuestos al cambio por culpa de su mentalidad comerciante. Para llevar una empresa industrial de forma eficiente, debes privilegiar el largo plazo y no tanto las ganancias inmediatas, como lo hacen las personas dedicadas a comprar y vender.

Los industriales se atribuyen como virtudes la creatividad, dar buenos trabajos y ser perseverantes. Un empresario industrial está en su fábrica, no en una oficina. Además, serían muy creativos y emprendedores, con capacidad de adaptarse a distintos contextos económicos.

Por otro lado, según otros empresarios el industrial ha sido caricaturizado como proteccionista, que hacía el menor trabajo posible, sobreprotegiendo su pequeño nicho de mercado porque solo le importaba su interés y ganar a costa de otro. Todos se valían del mismo discurso entorno a proteger a la industria nacional, aunque en realidad fuera protegerse a ellos mismos.

Después, al comerciante se le atribuye una visión de corto plazo y la búsqueda frecuente por aumentar el lucro. Ello porque constantemente se encuentra acostumbrado a comprar y vender, teniendo como meta encontrar márgenes de ganancia similares. Esta observación ha sido más frecuente por parte de los industriales más antiguos porque "muchos que ahora se llaman industriales nunca han dejado su mentalidad de comerciante". 
Otro grupo son los empresarios mineros, quienes consideran su labor fundamental en el desarrollo del país. Estos se consideran como agentes de progreso a sí mismos porque dan posibilidades de crecimiento a las zonas más alejadas del país. De esa forma, "nosotros vamos a la sierra, donde está la verdadera pobreza". Sin embargo, desde su punto de vista, este no comunica a pesar de tener muchas cosas a favor: da buenos trabajos, paga impuestos enormes y trae desarrollo a la sierra.

Para el caso de los gremios, estos están compuestos por empresas y las empresas deben trabajar. Ya uno dedica suficiente tiempo a resolver problemas que proponía el Estado para estar hablando con todo el mundo. En ese sentido, la representación de estos se refiere a un grupo que está dedicado a defender exclusivamente sus intereses.

En el caso de los gremios empresariales, estos se retratan como clubes de amigos en donde se encuentran los empresarios. Más que los gremios, son los estudios de abogados quienes imponen agendas porque se encargan de agrupar empresas y sacar leyes adelante. Además, otra característica de estos reside en la unidad de sus miembros: todos se conocen y forman un "espíritu de cuerpo".

La dirección de un gremio puede ser una labor muy complicada y para ello, existen las gerencias. Ahí uno actúa como bisagra, pudiéndose llegar cierto tipo de equilibrio cuando uno trabaja con independencia. El punto estaría en tratar de llegar a decisiones objetivas: esto es lo correcto y esto no es lo correcto. Regularmente, cuando hay rivalidades, mencionan que unos actúan por principios mientras que el otro defiende intereses solamente.

A nivel general, entonces, los acuerdos dentro de un gremio son "consensos dulcemente forzados". Esto porque uno debe ubicarse primero en dónde está: es decir, ser consciente de las necesidades de todos. Entonces, la labor desempeñada debe tomar en cuenta a las empresas peruanas y las internacionales, hacer propuestas que representen el bien común.

En conclusión, la principal característica del empresario es su capacidad de adaptarse al contexto y generar riqueza. Después, las representaciones junto con las formas de interacción se encontrarían explicadas si uno trabaja en un determinado sector. Es por ende que apreciamos un relato lleno de variedades y fisuras, que no son un único bloque. Luego, los gremios serían espacios donde forman un espíritu de cuerpo de cara al resto de la sociedad, generador de solidaridades. 


\section{Movimientos sociales y sociedad civil}

Otros grupos que fueron mencionados en las conversaciones han sido muy variados, van desde las colonias extranjeras hasta movimientos sociales como sindicatos y asociaciones campesinas. Esto se explica por la historia particular de cada uno, donde establece una determinada interacción y, con ello, determinadas representaciones. Así, el industrial describe mucho mejor al sindicalismo que un comerciante mientras que un minero conoce mejor a las comunidades campesinas que un banquero.

Primeramente, están las colonias extranjeras. De acuerdo con sus descendientes, estas pudieron integrarse a través de su trabajo y sus valores. Para los ítalo-peruanos, la inmigración fue muy positiva para el Perú porque muchos se integraron a la vida nacional y lograron triunfar. En el caso de los descendientes de alemanes, valores como la honestidad y la rectitud está presentes generación tras generación.

Por otro lado, los grupos de la sociedad civil más mencionados refieren a movimientos sociales con quienes más han interactuado en su vida cotidiana los empresarios como sindicatos y asociaciones campesinas. Después, todos mencionan a organizaciones como ONG pero desde posiciones muy ambiguas: van desde la admiración hasta el menosprecio. Además, hay una representación ambivalente respecto a los académicos.

La relación con los trabajadores ha cambiado, antes eran más fuertes como todas las organizaciones sociales. Lo más típico es recordar sindicatos protegidos por el Estado, muy politizados, con tensiones y huelgas. Sin embargo, con el paso del tiempo uno debería ir educando a los sindicalistas porque al final es de interés común que la empresa subsista porque cuando uno trata bien y paga bien a la gente no hay problemas sindicales.

Existe la idea de que los problemas laborales surgen si no hay un trato adecuado y un pago justo. Son muy temidos los conflictos laborales porque a nadie le gusta trabajar con problemas. En ese sentido, casi el compromiso que dicen tener con los empleados está escrito en piedra en el sentido que tratar mal al empleado puede considerarse tabú en el discurso.

Los más viejos recuerdan malas relaciones al inicio pero en la vida cotidiana, uno debía ser muy fino para tener buena relación con los trabajadores. En el caso de los más jóvenes, existe la representación del sindicato como un estorbo puesto que no permite tomar mejores decisiones. Esto se produce porque, con el neoliberalismo, las negociaciones colectivas han desapareci- 
do de tal forma que los gerentes más jóvenes simplemente no saben cómo hablar con el resto de empleados.

Después, son los mineros quienes ven a las comunidades campesinas como quienes han sufrido el abandono del Estado y muchas veces están manipulados por dirigencias, produciéndose falsas expectativas. Pueden ser muy duros para negociar y sus problemas internos son fuertes, por eso es difícil negociar con ellos. Por ende, muchos se apoyan en su ignorancia.

Esa dureza al momento de negociar es explicada porque son manipulados por gente que se aprovecha de ellos, usándolos como "carne de cañón”. En ese sentido, la posición más crítica sobre las ONG las ve como mecanismos para buscarse empleo, siendo poco profesionales y viviendo del resentimiento de las personas. Sin embargo, una posición más amable informa su rol en acompañar el liderazgo del Estado en la búsqueda del desarrollo, siendo agentes de progreso.

Los conflictos que se desarrollan entre empresas y sociedad civil a menudo han girado en torno a temas laborales y más recientemente a conflictos socio ambientales. En ese sentido, todos enfatizan en no generalizar; es decir, son algunos empresarios y ejecutivos quienes tienen conflictos, mientras que la gran mayoría no. Muchas veces se piensa que existen personas que azuzan los conflictos y se aprovechan de la gente que no está debidamente informada, pero también reconocen la responsabilidad de uno en el sentido de errores de "forma, más no de fondo".

En el caso minero, se generan falsas expectativas en la población. De acuerdo con muchos, las ONG desconocen la dinámica del mercado y es difícil comprender que las empresas son actores claves para el desarrollo del país. Creen que ellos los ven como los "malos de la película" o como un grupo de millonarios insensibles que solamente piensan en hacer dinero.

Respecto a la academia, existe cierto respeto a la labor de investigación que realiza. Es decir, ser una persona con títulos tienen un halo de legitimidad que enfatiza el conocimiento, haciéndose la comparación con un artista: una persona que no tiene ambiciones materiales pero que su labor es importante porque brinda cosas que hacen pensar a la sociedad. En ese sentido, un intelectual es una persona que debe respetarse: si bien no tendría el mismo dinero que uno, tiene conocimientos y educación que lo hacen "un igual".

Sin embargo, también hay otra imagen. Este tiene que ver con el tema de utilidad, bajo la premisa de que quienes se han dedicado a este tipo de actividades tienen envidia del mundo ejecutivo porque a pesar de los cono- 
cimientos no ganan lo mismo. De esa forma, la militancia política de muchos intelectuales parte del resentimiento social hacia el mundo corporativo.

Apreciamos entonces que las representaciones encontradas son muy ambivalentes. Para el caso del sector público, la imagen predominante es la del burócrata: indolente, corrupto e ineficiente al momento de realizar las cosas. De otra forma, el mundo ejecutivo tiene todas las características opuestas: práctico, eficiente y que brinda beneficios para la sociedad. Por último, el consenso general sobre otros grupos sociales es que no llegan a comprender y valorar la importancia de las empresas en el bienestar de la sociedad, siendo prejuzgados como personas que solamente piensan en dinero.

\section{Conclusiones}

En primer lugar, las representaciones surgen de la interacción de los grupos empresariales dentro de sus asociaciones. Cuando empresarios se juntan con empresarios, producen imágenes sobre otros actores, atribuyéndoles ciertas conductas. Esto porque estas instituciones constituyen una matriz sobre donde se perciben como grupo y forman solidaridades. Debemos recordar que estas representaciones no son homogéneas, sino que existen fisuras propias de las particularidades de cada entrevistado. Es decir, un relato no es sólo un bloque sino una amalgama de ideas articuladas en torno a una mayor.

Después, todas estas representaciones están estructuradas de acuerdo a caracterizaciones. Estas forman marcos de referencia que indican las conductas que pueden tener determinados individuos, con el propósito de brindar seguridad sobre una acción a futuro. Si bien mencionan que el objetivo no es generalizar, esta es asumida en la vida cotidiana como "regularmente es asî".

Por otro lado, las representaciones cumplen la función de diferenciar. Es por medio de las caracterizaciones que los entrevistados pueden identificar sus propios atributos y los ajenos para contar con herramientas que guían su interacción. En ese sentido, a diferencia de las ideologías nacionalistas clásicas donde el fin es integrador, bajo el neoliberalismo la función es separar a los grupos sociales en este sentido: "todos somos peruanos, pero ustedes no son igual a mí porque todos viven de mi trabajo".

De acuerdo con todo lo descrito, existe una representación muy negativa del Estado. Esta desconfianza tiene su origen en el último gobierno militar (1968-1980) donde hubo bastante "hostilidad" al sector privado producto de 
las estatizaciones. Ello se vio más reforzado todavía por la emergencia del pensamiento neoclásico en el manejo económico, consolidándose durante toda la década del noventa. Es decir, el Estado es poco práctico, corrupto e indolente con las necesidades de los demás.

Respecto al mundo corporativo, los empresarios y las empresas son representados como quienes generan trabajo, son luchadores y están acostumbrados a solucionar problemas permanentemente, que se arriesga a apostar verdaderamente por su país porque pone su capital a disposición del resto. Sin embargo, creen que el resto de la sociedad los ve como insensibles, ambiciosos y déspotas.

Por otro lado, la representación de otros grupos es muy ambigua. Puede ir desde el respeto (academia, sindicatos) hasta el desprecio (movimientos sociales, grupos de izquierda) aunque consideran que la sociedad no comprende o valora el rol que cumplen los empresarios en la generación de riqueza. De esa manera, consideran que su rol es importante porque, de cierta forma, ellos "mantienen" a toda la sociedad peruana. Es por medio de esta representación que buscan justificar un trato privilegiado.

Finalmente, podemos concluir que las representaciones son parte de las formas en como las elites económicas han constituido la nación desde la década de los noventa. Así, la influencia más importante proviene del neoliberalismo, que se ha ido introduciendo en la mentalidad de ejecutivos y empresarios como un sentido común. Por otro lado, este se mezcla con un sentimiento de pertenencia donde todos se creen y se identifican con el país porque han nacido acá y porque la idea de nación ha sido inculcada por su familia a lo largo de su vida. Este sería un país bello, con muchas condiciones para salir adelante y donde una persona que de verdad ama a su país se queda para pelear desde adentro creando cosas.

En síntesis, puede apreciarse que la mayoría de los entrevistados conciben la nacionalidad peruana sobre la base de un liberalismo criollo, esto es, la adaptación del pensamiento neoliberal clásico al contexto peruano. Este sostiene que una comunidad se encuentra compuesta por individuos que para progresar deben enriquecerse, siendo la única posibilidad de "salir adelante" el trabajo productivo dentro de un mercado libre. En ese sentido, los empresarios son quienes generan riqueza y empleo, dando auténtico bienestar a las personas y mereciendo un trato privilegiado. Es dentro de esta forma de concepción donde surgen las representaciones de otros actores con el fin de desarrollar un proyecto político que sea capaz de aglutinar a todos los que se identifican como peruanos. 


\section{Bibliografía}

Alcorta, L. (1989) Concentración y centralización de capital en el Perú. Lima: Fundación Friedrich Ebert.

Anaya, E. (1990). Los grupos de poder económico: un análisis de la oligarquía financiera. Lima: Editorial Horizonte.

Anderson, B. (1993). Comunidades imaginadas: reflexiones sobre el origen y difusión del nacionalismo. México D.F.: Fondo Cultura Económica.

Balibar, E. (2003). Nosotros, ¿ciudadanos de Europa?: las fronteras, el Estado y el pueblo. Madrid: TECNOS.

Bourdieu, P. (2011). Las estrategias de la reproducción social. Buenos Aires: Siglo XXI.

Burga, M., \& Flores Galindo, A. (1980). Apogeo y crisis de la república aristocrática. Lima: Ediciones Rikchay.

Campodónico, H., \& Durand, F. (2018). Poder empresarial y sociedad civil en Sudamérica. Lima: DESCO.

Chatterjee, P. (2002). Comunidades imaginadas: ¿por quién? Historia Caribe, 7(2), 43-52.

COMEX PERÚ (2010). Negocios Internacionales. Año XX.

Cotler, J. (1978). Clases, Estado y Nación en el Perú. Lima: IEP.

Durand, F. (2016). Cuando el poder extractivo captura el Estado: lobbies, puerta giratoria y paquetazo ambiental en el Perú. Lima: OXFAM.

García-Huidobro, R. (2016). La narrativa como método desencadenante y producción teórica en la investigación cualitativa. EMPIRIA: Revista de Metodología de las Ciencias Sociales. 34(1),155-178.

Hobsbawn, E. (1997). Naciones y nacionalismo desde 1780. Barcelona: Crítica.

Kogan, L. (1999). Relaciones de género en las familias de sectores altos de Lima. Debates en Sociología, 23, 191-208.

Malpica, C. (1964). Los dueños del Perú. Lima: Editorial PEISA.

Palti, E. (2003). La nación como problema: los historiadores y la cuestión nacional. Buenos Aires: Fondo Cultura Económica.

Ramos, L. (2014). Redes de lobby informal de los gremios empresariales frente a la administración pública del Ministerio de Economía y Finanzas del Perú al momento de debatir la propuesta de la Ley General del Trabajo (2011-2012). Tesis PUCP.

Rénique, J. L. (2008). Esperanza y fracaso en la historia del Perú. En: PASARA (Ed.). El Perú en el siglo XXI. Lima: PUCP. 
Reyes, A. (2008). Calixto Romero: para quitarse el sombrero. Palmas del Espino S.A. Lima.

Wallerstein, I. (2006). Abrir las ciencias sociales: informe de la comisión Gulbenkian para la reestructuración de las ciencias sociales. México DF.: Siglo XXI Editores.

Fecha de envío: 2019/05/25; Fecha de aceptación: 2019/08/08;

Fecha de publicación: 2019/09/01 